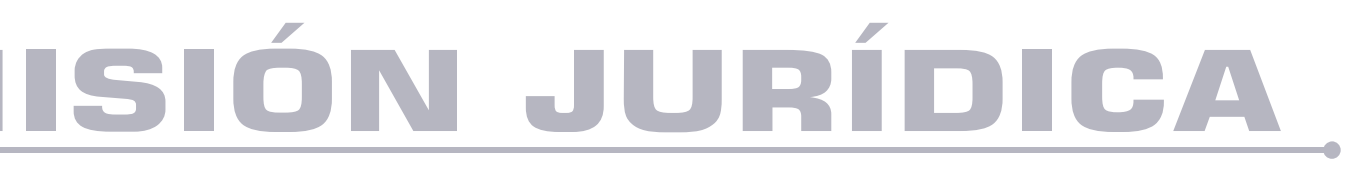

\title{
La construcción del Otro en el discurso de las constituciones colombianas: Una lucha por el reconocimiento y la responsabilidad frente al Otro
}

The construction of the Other in the discourse of Colombian

Constitutions: A struggle for the recognition and responsibility against the Other

Autor: Jafeth Paz Rentería

DOI: https://doi.org/10.25058/1794600X.1053

\footnotetext{
Ś MISIÓN JURÍDICA A
} 


\title{
LA CONSTRUCCIÓN DEL OTRO EN EL DISCURSO DE LAS CONSTITUCIONES COLOMBIANAS: UNA LUCHA POR EL RECONOCIMIENTO Y LA RESPONSABILIDAD FRENTE AL OTRO*
}

\author{
The construction of the Other in the discourse of Colombian \\ Constitutions: A struggle for the recognition and \\ responsibility against the 0 ther \\ A construção do outro no discurso das constituições \\ colombianas: uma luta por reconhecimento e \\ responsabilidade em relação ao outro
}

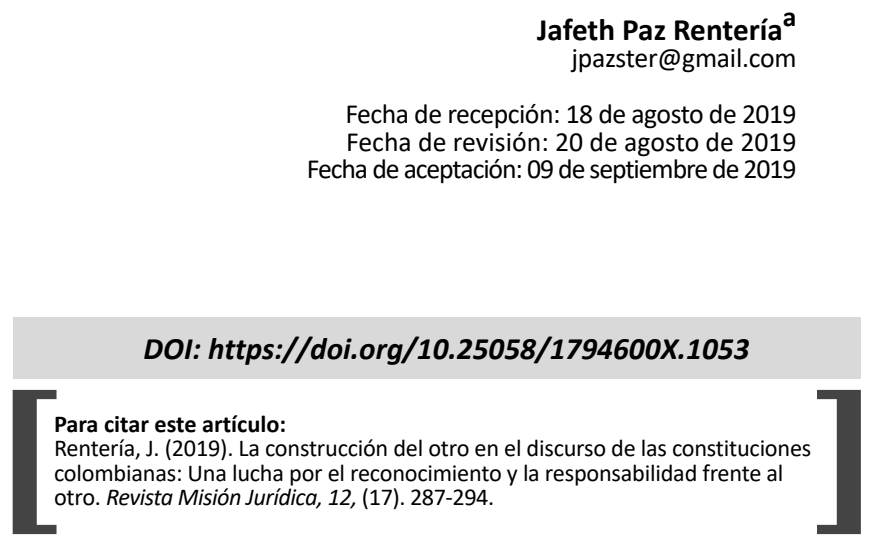

\section{RESUMEN}

En este artículo se hace un recorrido de la lucha y las tensiones por el reconocimiento del Otro en el discurso de las constituciones nacionales colombianas, en una etapa histórica caracterizada por la captura conceptual directa del Estado, la cual derivó en que los dispositivos constitucionales y los demás materiales del ordenamiento jurídico funcionaran desde la lógica de la exclusión.

Así, este proceso de construcción del Otro tuvo como elementos estructurantes cuatro categorías determinantes: La inferiorización, mediante la cual la mayoría de la población fue desplazada de los espacios de poder y decisión; la invisibilización, que permitió representar el colectivo exclusivamente desde la perspectiva eurocentrica; la cosificación, con la que el Otro extraño y diferente fue subhumanizado y la instrumentalización, que validó el lugar de las mujeres y los pobres en función de los intereses del capitalismo y de la sociedad patriarcal.

\footnotetext{
*Artículo de reflexión

a. Magister en Derecho Administrativo de la Universidad del Rosario. Especialista en Derecho Laboral y Seguridad Social de la Corporación Universitaria Republicana. Abogado graduado con honores de Uniciencia. Periodista de la Fundación Universitaria Los Libertadores y Licenciado en Teología de la Fundación Universitaria San Alfonso. Docente del departamento de derecho de la Universidad Central y en posgrados de la Universidad La Gran Colombia. Estudios de doctorado en Filosofía en la Pontificia Universidad Bolivariana.
} 
Sin embargo, a partir de 1991 con el proyecto de una sociedad abierta y pluralista se emprendió, al menos formalmente, la construcción de un nuevo Otro desde la solidaridad, la dignidad humana y la responsabilidad. Pero desde ese momento, este ideal constitucional se ha enfrentado a desencuentros, tensiones y contradicciones ante los patrones ya instalados en el sistema social desde el régimen constitucional anterior.

Ante esto, el desafío de la filosofía de la responsabilidad de Emmanuel Levinas, el pensamiento de los pueblos originarios y de otros autores puede marcar un horizonte ético que sirve para desestructurar las diversas manifestaciones de la lógica de exclusión y sus instrumentos operativos que se reproducen continuamente en los imaginarios colectivos.

\section{PALABRAS CLAVES}

Lógica de exclusión, construcción del Otro, filosofía de la responsabilidad y captura conceptual del Estado.

\section{ABSTRACT}

In this article, a journey is made of the struggle for the recognition of the Other in the discourse of the Colombian constitutions, in a historical stage characterized by the direct conceptual capture of the State, which is derived in the constitutional devices and other materials of the ordering Legal functions from the logic of exclusion.

Thus, this construction process also had structuring elements such as inferiorization, invisibility, reification and instrumentalization. However, starting in 1991 with the project of an open and pluralistic society, the construction of a new Other was undertaken, at least formally, from solidarity, human dignity and responsibility. But from that moment on, this constitutional ideal has faced the disagreements, tensions and contradictions against the political patterns that had been already installed in the social system since the previous constitutional regime.

Given this context, the challenge of the philosophy of responsibility of Emmanuel Levinas can mark an ethical horizon that serves to deconstruct the diverse expressions of the logic of exclusion and the instruments that are continuously reproduced in collective imaginaries.

\section{KEYWORDS}

Logic of exclusion, construction of the Other, philosophy of responsibility and conceptual capture of the State.

\section{RESUMO}

Este artigo descreve uma jornada de lutas e tensões pelo reconhecimento do outro no discurso das constituições nacionais colombianas, numa etapa histórica caracterizada pela captura conceitual direta do Estado, que resultou em dispositivos constitucionais e outros materiais do sistema jurídico funcionarão a partir da lógica da exclusão.

Assim, esse processo de construção do outro teve como elementos estruturantes quatro categorias determinantes: A interiorização, pela qual a maioria da população foi deslocada dos espaços de poder e decisão; a invisibilidade, que permitiu representar o coletivo exclusivamente da perspectiva eurocêntrica; a reificação com a qual o outro estranho e diferente foi subumanizado e a instrumentalização, que validava o lugar das mulheres e dos pobres de acordo com os interesses do capitalismo e da sociedade patriarcal.

Contudo, a partir de 1991, com o projeto de uma sociedade aberta e pluralista, foi empreendida, pelo menos formalmente, a construção de um novo outro da solidariedade, dignidade e responsabilidade humana. Mas desde então, esse ideal constitucional tem enfrentado desacordos, tensões e contradições diante dos padrões já instalados no sistema social desde o regime constitucional anterior.

Diante disso, o desafio da filosofia de responsabilidade de Emmanuel Levinas, o pensamento dos povos indígenas e de outros autores pode marcar um horizonte ético que serve para reestruturar as várias manifestações da lógica da exclusão e seus instrumentos operacionais que se reproduzem continuamente no imaginário coletivo.

\section{PALAVRAS-CHAVE}

Lógica de exclusão, construção do Outro, filosofia de responsabilidade e captura conceitual do Estado. 


\section{INTRODUCCIÓN}

El proceso de construcción del Otro en lo que hoy es Colombia ha estado marcado por hitos constitucionales que, indudablemente, han dejado huellas sociales que siguen presentes en los comportamientos cotidianos. Las dinámicas de múltiples formas de violencia, entre ellas las violencias simbólicas ${ }^{1}$, y la luchas por el reconocimiento del $\mathrm{Otro}^{2}$, considerado por el conocimiento hegemónico como extraño, lejano y "salvaje", tienen una explicación en lo que fue la legitimidad pasada que se fundamentó en los regímenes constitucionales anteriores, formalmente reemplazados por la Constitución de 1991.

En este orden se ideas se puede considerar que a partir de la configuración constitucional actual estamos en la etapa de construcción de una alteridad que dio un giro respecto a la aproximación y concepción de lo Otro que, en siglos pasados, se había edificado desde la lógica de la exclusión. Sin embargo, este proceso no ha sido pacífico, sino que es un producto de desencuentros, tensiones $y$ contradicciones entre los imaginarios colectivos consolidados de rechazo al Otro, por diferente y extraño, y la nueva legitimidad inaugurada desde el núcleo del Reconocimiento al Otro.

Este marco permite una aproximación iusfilosófica en la construcción del Otro, que nos permite sostener como tesis que, en el caso colombiano, ha operado una "captura conceptual del Estado"4 desde los dispositivos constitucionales que instalaron lo que denomino una "lógica de exclusión", de la cual emergió un sistema de relaciones intersubjetivas de rechazo, alejamiento, irresponsabilidad e inferiorización del Otro.

1. La noción de violencia simbólica fue acuñada por Pierre Burdieu en 1970. Sobre el tema véase Fernández (2005).

2. Para profundizar en el tema véase Prada Londoño, 2017.

3. Es importante tener en cuenta que la Ley 89 de 1890, aún vigente en el ordenamiento jurídico colombiano, calificada como una de las tantas leyes de la vergüenza nacional, categorizó oficialmente a los indígenas como "salvajes" hasta el año de 1996, cuando mediante la Sentencia C-139 de 1996 M.P. Carlos Gaviria Díaz, la Corte Constitucional declaró inexequible esta expresión producto de un contexto histórico construido desde una "concepción ética universalista que consideraba lo diferente como incivilizado".

4. El Estado en su operación ha respondido a la intención de materializar unos conceptos, que se expresan en las características de los sistemas normativos. Al respecto véase Paz, 2014.
A partir de algunas premisas conceptuales sostenidas por Emmanuel Levinas (2011), se considera que el marco constitucional actual genera unos retos que implican la deconstrucción de lo Otro con el fin de desaprender lo aprendido, para así generar nuevos procesos de relacionamiento y de intersubjetividad que tienen como barreras en el mundo social -ya no en la juridicidad- la reproducción y materialización permanente de los imaginarios y el marco de relaciones jerarquizadas, en las que la clave teórica es la inferiorización, la invisibilización, la cosificación y la instrumentalización del Otro. En términos de Levinas esta situación demanda un proceso de humanización en el que "el humanismo solo debe ser denunciado porque no es suficientemente humano" (2011: 127).

Para lograr este propósito argumentativo el artículo tematiza la construcción del Otro en los regímenes constitucionales anteriores a 1991, reflexiona sobre el proyecto de construcción del Otro en la Constitución Política de 1991 y, haciendo énfasis en lo planteado por Levinas, analiza una construcción de la alteridad desde la responsabilidad.

\section{La construcción del Otro en los regímenes constitucionales anteriores a 1991}

Las constituciones, en tanto son proyectos colectivos de una sociedad en un momento humano determinado, no solo contienen el catálogo de derechos que se garantiza a los ciudadanos, la distribución de competencias entre los diferentes órganos del Estado o la estructura y organización interna del mismo. También son discursos que se construyen para garantizar la legitimidad de un momento histórico determinado y son el reflejo del sistema de creencias de quienes en la realidad objetiva ostentan lo que Lassalle (2012) denominó "factores reales del poder".

En tanto discurso "hijo de su tiempo", la Constitución funciona como sintetizadora del pensamiento de quienes pretenden representar a las sociedades políticas organizadas estatalmente. Por esta razón, desde el discurso directo y subyacente de la Constitución, se pueden inferir las claves teóricas que explican profundamente los dispositivos normativos, que a su vez definen

5. El historiador francés Marc Bloch dijo que "los hombres no son tan hijos de sus padres como de su tiempo". 
las políticas de relaciones intersubjetivas en la sociedad y las lógicas operativas de dicho proceso.

En este sentido, desde la postura iuspositivista el análisis de los horizontes interpretativos que se desprenden de las constituciones tienen un enfoque marcadamente normativo, que es problemático porque así se invisibilizan las aproximaciones fenomenológicas, el aparato conceptual y el conjunto de relaciones internas que emergen cuando se realiza una aproximación teórica a las constituciones como discurso.

Por esta razón se puede considerar que el discurso de carácter constitucional es la expresión política y la juridificación de la manera como se configura un horizonte legitimador de aproximación a los otros, que interviene como fundamento formal de las relaciones $y$ jerarquizaciones sociales $\mathrm{y}$, de paso, contiene la autodefinición diferenciadora de los sujetos dominantes, que se asumen en el mundo de la vida (Habermas, 2002) desde la definición arbitraria de aquellos otros excluidos de los espacios de poder y de los procesos que moldean y configuran a las sociedades y al Estado.

Esto quiere decir que el texto constitucional como discurso, contiene en sí un proceso hermenéutico del Otro, que ya está preconcebido socialmente $\mathrm{y}$, por lo general, los elementos estructurantes de esta interpretación, tienen las huellas de los prejuicios y concepciones teóricas que se instalan en las constituciones como determinadores de las múltiples lecturas que hace la sociedad de su propia realidad y de su régimen constitucional. Esto es lo que denomino "captura conceptual del Estado" en su expresión discursiva constitucional.

Ésta idea de captura conceptual se expresa en los discursos que tienen una función legitimadora, como es el caso de los textos constitucionales y en general de los ordenamientos jurídicos, los cuales tienen una dimensión que se objetiva y materializa en las relaciones intersubjetivas, institucionales y en la cotidianidad. Igualmente, esta captura implica que la razón de ser del Estado y de todas las instituciones creadas a partir de éste, se encuentran orientadas y dominadas por los conceptos que introducen directa y sofisticadamente los detentadores del poder en el aparato constitucional, como una forma de direccionar y ajustar la realidad social y las relaciones con aquellos sujetos otros desde el marco de sus intereses.
En un primer momento este proceso de captura se manifiesta de manera directa (Paz, 2014) y se expresa mediante las diversas concepciones de lo que categorizo como la lógica de la exclusión, en tanto otorga privilegios a los sectores dominantes, mientras instrumentaliza e invisibiliza a la gran mayoría dominada.

En nuestro marco inicial de análisis, es decir los textos constitucionales que se expidieron en Colombia con anterioridad a la actual Constitución Política de 1991, la captura conceptual de orden excluyente se expresó con la idea de nacionalidad y ciudadanía que estaba circunscrita a las élites constructoras del discurso constitucional.

Por ejemplo, en la Constitución de 1821, aunque formalmente indicaba que el país no era patrimonio de ninguna familia o persona, expresamente limitaba la nacionalidad a los hombres libres y la capacidad de sufragar a los nacionales, casados o mayores de 25 años que supieran leer o escribir, tuvieran finca raíz o ejercieran algún oficio o profesión con casa o taller sin dependencia de otro ${ }^{6}$. De entrada estaba por fuera de este concepto la gran mayoría de la población, que en ese momento no sabía leer ni escribir (más del 90 por ciento de la población), no incluía a las mujeres, a los esclavizados y a quienes no tenían la capacidad económica de trabajar de manera independiente. Solo el 5\% de la población de ese momento cumplía dichos requisitos de ciudadanía.

Así el dispositivo constitucional contiene una definición de aquellos otros excluidos, construida a partir de la autodefinición de los privilegiados y gira alrededor de la concentración del poder en unos pocos de las decisiones trascendentales del Estado. A partir de estos contenidos que demuestran la "lógica de exclusión", se puede ubicar el lugar de enunciación desde el cual los constructores de los textos constitucionales nos permiten escuchar sus voces respecto a los otros considerados como extranjeros en su territorio, como humanos subhumanos y humanos no humanos, a los que se les cierra la puerta en el poder decisiorio y en el derecho a entrar en el juego político de la sociedad.

6. "Artículo 21.- Para ser elector se requiere: 1 . Ser sufragante no suspenso; 2. Saber leer y escribir; 3. Ser mayor de veinticinco años cumplidos y vecino de cualquiera de las Parroquias del Cantón que va haber las elecciones; 4. Ser dueño de una propiedad raíz que alcance el valor libre de quinientos pesos, o gozar de un empleo de trescientos pesos de renta anual, o ser usufructuario de bienes que produzcan una renta de trescientos pesos anuales, o profesar alguna ciencia o tener un grado científico". Constitución Política de 1821, Art. 21 
Esta aproximación al Otro desde la inferiorización y la invisibilización con diversos matices, será una huella constante que en el futuro orientará el discurso constitucional y el ordenamiento jurídico colombiano antes de la Constitución de 1991. Entonces el centro de gravedad de la mirada y del proceso de relacionamiento con el Otro no contiene una "llamada" que demande una respuesta a la manera de la "lógica de la seducción" que sostiene Jean-Luc Marion $^{8}$ y por el contrario, en el discurso se impone una cosificación e instrumentalización jurídica y en el discurso oficial del Otro, que es silenciado para que no responda a su lugar en la historia.

Más adelante la Constitución de 1830, con la que se quiso superar la crisis centralista que había generado la Constitución de 1821 y que en la práctica solo rigió por unos meses -porque lo que hoy es Venezuela y Ecuador se separaron del proyecto de La Gran Colombia- mantuvo la misma constitución de lo Otro y del Otro desde la invisibilización y la exclusión ${ }^{9}$, aunque por la denominada libertad de vientres se incluyó a los libertos nacidos en Colombia en la condición de nacionales, pero en el papel. Sin embargo, en los derechos políticos siguieron por fuera los analfabetos, quienes no tuvieran propiedad raíz y no ejercieran una profesión sin sujeción a otro en calidad de sirviente doméstico o jornalero. Es decir, en esencia el marco de las relaciones institucionalizadas en el discurso constitucional entre las élites y aquellos otros subalternizados siguió capturada por la idea de superioridad que marginó a la mayoría de la población de sus derechos políticos.

Ésta relación asimétrica con el Otro se estructuró progresivamente a partir de teorías teológicas y jurídicas que pretendieron justificar los procesos de conquista y de colonización en todo el mundo. En efecto, y aunque la organización del Estado encuadrado en una Constitución se

7. Según Marion "seducir consiste de entrada en hacerle admitir al. Otro, no que lo he seducido, sino que he intentado seducirlo y que él me ha dejado intentarlo -tal es la respuesta que ratifica que una llamada ha sido lanzada."

8. Marion, J., 2008.

9. "Artículo 14.- Para gozar de los derechos de ciudadano se necesita: 1. Ser colombiano; 2. Ser casado o mayor de 21 años; 3 . Saber leer y escribir; pero esta condición no será obligatoria hasta el año de 1840; 4. Tener una propiedad raíz, cuyo valor libre alcance a trescientos pesos, o en su defecto ejercer alguna profesión o industria que produzca una renta anual de ciento cincuenta pesos, sin sujeción a otro, en calidad de sirviente doméstico o jornalero". (Constitución Política de 1830). ha considerado como uno de los logros de la Modernidad y del pensamiento de la Ilustración, es evidente que el discurso estructurado comprendió el principio de igualdad, pero solo circunscrito a los intereses de la élite dominante. Por lo consiguiente, aquellos otros que constituían a la mayoría de la población, no estaban incluídos en esa idea de igualdad y libertad con la que se justificaron las revoluciones.

Las otras constituciones del siglo XIX de lo que hoy es Colombia, esto es, la Constitución de la Nueva Granada de 1832, de 1843, 1848, 1853, la Confederación Granadina de 1858 y la de 1886 siguieron marcando el horizonte de lo Otro y del Otro desde la lógica de la exclusión que estructuró un aparato estatal y un mundo social que legitimó el racismo, la discriminación de los otros, la cultura patriarcal y el monismo religioso, cultural y jurídico. La Constitución de los Estados Unidos de Colombia de 1863 fue un discurso que se salió de esta óptica $y$, posteriormente las reformas que periódicamente se fueron realizando a la Constitución de 1886, de una u otra manera, fueron dando un giro en el lento proceso de desmonte de este horizonte de exclusión, que formalmente se desestructuró con la actual Constitución de 1991.

Sin embargo, el reconocimiento teórico del Otro y de todas sus expresiones en el actual discurso constitucional está en tensión constante con el moldeamiento del mundo social que sigue funcionando desde la lógica anterior. Es decir, tenemos una nueva definición del Otro y de los Otros, que dio un giro de 180 grados en los contenidos constitucionales de 1991, los cuales están en oposición con el sistema de creencias y los imaginarios colectivos de configuración del Otro, que siguen instalados en la cultura, en la forma de pensar y en la institucionalidad.

Esta situación ha creado un campo de tensión entre el carácter ideal de lo consagrado en la Constitución y la realidad social que sigue funcionando con los núcleos sociales excluyentes que legitimó el régimen anterior.

\section{El Otro en la Constitución de 1991}

El giro conceptual del discurso constitucional actual tiene como elemento estructural esencial 
el principio del pluralismo ${ }^{10}$ en la concepción del Estado y el reconocimiento y protección de los Otros en su diversidad étnica y cultural ${ }^{11}$. De esta manera, contrario a los régimenes constitucionales anteriores el constructo del ser nacional y de los derechos de ciudadanía no tienen las restricciones configurativas del pasado. Es decir, estamos ante un nuevo ideal de proyecto colectivo de nación caracterizado por el multiculturalismo y la interculturalidad, el cual supera el monismo anterior y filosóficamente se fundamenta en el Reconocimiento del Otro, en los términos de Paul Ricoeur ${ }^{12}$.

En este orden de ideas, en la Constitución de 1991 emergieron expresamente sujetos colectivos de derechos con un amplio ámbito de movilidad en el escenario estatal, partiendo de sus propias cosmovisiones, lenguas oficiales en sus territorios, etnoeducación, derecho propio para la solución de sus controversias y autonomía en la administración de sus intereses colectivos. ¿Esto significa que los procesos de captura descritos anteriormente han terminado? ¿El reconocimiento formal e institucional del Otro se ha traducido en la terminación de la lógica de exclusión del régimen anterior?

Aunque ésta es una inquietud que no atiende el propósito de este trabajo, se debe anotar que además de la captura conceptual del Estado, de la se habló anteriormente, en el caso colombiano, históricamente, se han desarrollado otros procesos que el autor denomina captura directa, indirecta y sofisticada ${ }^{13}$. Y hoy, cuando la lucha por el reconocimiento desde la formalidad jurídica ha dejado de ser un campo de tensión, al menos en lo que tiene que ver con la universalidad de los derechos políticos, la captura sofisticada del Estado como una estrategia neutralizante del derecho, es un fenómeno que posibilita la explicación de la ineficacia y falta de goce efectivo de los derechos que se reconocen en el ordenamiento jurídico.

En cuanto al reconocimiento del Otro, si bien es cierto que desde el discurso constitucional ya se tiene una garantía formal, no se puede desconocer

\footnotetext{
10. Constitución Política, Art. $1^{\circ}$

11. Constitución Política, Art. 7ํㅗ

12. Para una profundización sobre este tema véase Prado (2017).

13. Estas categorizaciones se han trabajado en Paz, 2014
}

que hay un choque permanente con la realidad que sigue permeada por la lógica de la exclusión. Entonces el gran desafio es la materialización de ésta constitución del Otro que parte de su reconocimiento desde las dimensiones de la pluralidad, la diversidad y la responsabilidad.

\section{La construcción del Otro desde la responsabilidad}

Desde el enfoque de la captura conceptual del Estado se puede analizar que el edificio estructural de la construcción del Otro en Colombia ha tenido intencionalidades que se han concretado en los elementos característicos de la lógica de la exclusión, que se ha definido como inferiorización, invisibilización, cosificación e instrumentalización.

Inferiorización porque desde esa lógica la relación con el Otro no se estructuró desde un plano horizontal, sino vertical, de arriba hacia abajo. Invisibilización porque los principales espacios de poder fueron ocupados por una idea de nación excluyente y una ciudadanía censitaria, y cosificación e instrumentalización porque aquellos excluidos por la cultura y la cosmovisión hegemónica, solo tuvieron un cierto nivel de importancia en tanto sirvieran a los intereses de la minoría élite.

Ésta aproximación al Otro derivó en el "invento" de un nuevo mundo a través de la configuración de un "palimpsesto cultural"14 que se dio a partir de un proceso que consistió en la actividad de "borrar" y "reescribir" otros mundos y conocimientos posibles, desde una posición de superioridad e irresponsabilidad frente lo Otro y los otros.

Esta realidad que se puede considerar como un epistemicidio, término trabajado a profundidad por de Sousa Santos (2010), en cuanto negación del Otro como productor de conocimiento valido para un contexto occidental. Como experiencia humana nos invita en la actualidad a la construcción permanente del Otro, desde un sentido de responsabilidad y de desinterés, tal como lo dice Levinas: "Instauración de un ser que no es para sí, que es para todos, que es a la vez ser y desinterés; el para sí significa conciencia de sí; el para todos significa responsabilidad para con los otros, soporte del universo." (Levinas, 2011)

14. Palimpsesto significa "grabado nuevamente". En los manuscritos hace referencia a aquellos que conservan huellas de uno anterior. En la construcción cultural del país, ha pasado algo similar. 
Un ser que no es para sí, esto es, que no está en función de sus propios intereses, no se autodefine a partir de la definición limitada e inferiorizada del Otro, y, en conciencia de sí, está en función de todos con un sentido de responsabilidad que se traduce en una ética hacia el otro que le impide inferiorizarlo, invisibilizarlo, cosificarlo e instrumentalizarlo. En teoría, desde nuestra propuesta, esta es la dimensión aspiracional de la Constitución Política de 1991 cuando fundamenta el Estado Social de Derecho en los principios de dignidad humana y de solidaridad.

Pero como ya hemos advertido, la dinámica filosófica de la Constitución en este proyecto está en contravía con las relaciones vivas del sistema cultural que siguen reproduciendo las lógicas de construcción del Otro legitimadas en los regímenes constitucionales anteriores. Es decir, la constitución aspiracional con su carácter ideal va por un lado, mientras los imaginarios colectivos de la vida cotidiana por otro, generando de esta manera, dinámicas de enfrentamiento, múltiples violencias y la ineficacia de los derechos. Esto hace que la problemática actual no sea por la conquista de derechos, sino por su efectivización, matierialización y goce efectivo.

A demás de esto, no se puede desconocer que la misma constitución, en la interpretación que han privilegiado los gobernantes de turno, ha posibilitado unas contradicciones $\mathrm{y}$ tensiones entre los derechos de los sujetos colectivos reconocidos y un modelo económico y de desarrollo de corte neoliberal, en el que la hermenéutica del Otro y de lo Otro se lee desde el prisma de los intereses económicos de multinacionales y grupos de presión nacionales.

Ante este panorama, nuevamente Levinas y su filosofía de responsabilidad dejan un desafío que constituye un reto para que se haga realidad:

Ser sí mismo, de otro modo que ser, desinteresarse es cargar con la miseria y el fallo del otro e inconcluso con la responsabilidad que el otro puede tener respecto a mí. Ser sí mismo -condición de rehen- es tener siempre un grado de responsabilidad superior, la responsabilidad respecto a la responsabilidad del otro (...) [y con] una responsabilidad que me compromete antes de toda verdad y de toda certeza (Levinas, 2011).

\section{CONCLUSIONES}

La construcción de ese nuevo Otro "suficientemente humano" en términos de Levinas, es uno de los grandes desafíos de la humanidad en la actualidad porque conlleva a una ruptura y a una nueva lectura de muchos de los paradigmas sociales, filosóficos, jurídicos, políticos y religiosos que sirvieron de soporte conceptual a la Modernidad, al mundo Contemporáneo y a lo que algunos denominan Postmodernidad.

En lo subyacente esta propuesta también implica la redefinición y nuevo entendimiento del capitalismo, de cualquier otro sistema económico emergente, del mercado y de los nacionalismos dominantes que alejan a los seres humanos de la proximidad de aquel Otro humano que se desconoce y se rechaza.

Muchos de los desencuentros, tensiones y contradicciones de la actualidad se pueden explicar desde la captura conceptual del Estado como una clave teórica que descubre el juego de intereses que en el pasado se expresaba directamente en los dispositivos constitucionales y hoy, de manera sofisticada, afecta, neutraliza y "eclipsa" el proceso de materialización del derecho como una agenda de contracultura.

La dimensión de este reconocimiento del Otro, aunque en principio tiene alcances en el plano de las relaciones intersubjetivas, también tiene efectos de múltiple vía en la idea "otros", "otras", entendidos como sujetos colectivos que históricamente también han padecido el esquema excluyente de construcción de lo Otro.

Indudablemente este nuevo Otro configurado desde el "para todos"y el "des-interés" entendido como "responsabilidad para con los otros" tal como lo propone Levinas, en términos reales llega a ser una recreación del modo de vivir y del modo de relacionarnos como humanidad, que al expandirse y vincular a la naturaleza, constituyen el principio de acción de otro mundo posible. 


\section{BIBLIOGRAFÍA}

- Asamblea Nacional Constituyente. (1991). Constitución Política de Colombia 1991.

- Congreso de Cúcuta. (1821). Constitución de la República de Colombia de 1821

- Congreso de la República de Colombia. (1830) Constitución Política de Colombia de 1830.

- Congreso de la República de Colombia. (1890). Ley 89 de 1890, Por la cual se determina la manera como deben ser gobernados los salvajes que vayan reduciéndose a la vida civilizada.

- Corte Constitucional de Colombia. (1996). Sentencia C-139 de 1996 M.P. Carlos Gaviria Díaz.

- de Sousa Santos, B. (2010). Descolonizar el saber, reinventar el poder. Uruguay: Trilce.

- Fernández Fernández, J. M. (2005). La noción de violencia simbólica en la obra de Pierre Bourdieu: una aproximación crítica. Cuadernos de Trabajo Social, 18, 7 - 31.
- Habermas, J. (2002a), Teoría de la acción comunicativa. I: Racionalidad de la acción y racionalización social, 3a ed. Madrid: Taurus.

- Lasalle, F. (2012). ¿Qué es una constitución? Bogotá: Ariel.

- Levinas, E. (2011) De otro modo que ser o más allá de la esencia. Madrid: Ediciones Sígueme.

- Marion, J. (2008) Siendo dado. Ensayo para una fenomenología de la donación. Madrid: Editorial Síntesis.

- Paz Rentería, J. (2014) Afrocolombianos y el Territorio: Análisis desde la Teoría de la Captura del Estado. Bogotá: Editorial Ibañez.

- Prada Londoño, M. (2017). Entre disimetría y reciprocidad. El reconocimiento mutuo según Paul Ricoeur. Bogotá: Editorial Aula de humanidades y Universidad de San Buenaventura Bogotá. 